\title{
A STUDY OF THE MODE OF ACTION OF THE ADRENAL MEDULLARY HORMONES ON SODIUM, POTASSIUM AND WATER EXCRETION IN MAN ${ }^{1,2}$
}

\author{
By JAMES F. NICKEL,, 4 CHEVES McC. SMYTHE,3, 5 EMANUEL M. PAPPER, \\ AND STANLEY E. BRADLEY
}

(From the Departments of Medicine and Anesthesia, Columbia University College of Physicians and Surgeons, and the Presbyterian Hospital, New York, N. Y.)

(Submitted for publication July 8, 1954 ; accepted August 18, 1954)

The adrenal medullary hormones, $l$-epinephrine and $l$-norepinephrine, elicit differing systemic hemodynamic responses when administered in physiologic dosage to normal man $(2,3)$. Both produce an elevation in the arterial pressure but $l$-epinephrine does so by increasing cardiac output in association with diminished or unchanging peripheral vascular resistance, whereas $l$-norepinephrine usually induces a fall in cardiac output and an increase in resistance. Both evoke renal vasoconstriction and a reduction in renal blood flow (4-6) with a decrease in the urinary concentration and excretion of sodium and potassium $(7,8)$. Since glomerular filtration is not affected (5-9) the reduction in electrolyte output may be ascribed to augmented tubular reabsorption. The urine flow remains essentially unchanged or increases during intravenous infusion of either agent and usually declines abruptly upon termination of the pressor response.

These alterations in urine formation may be ascribed to a direct action of the adrenal medullary hormones upon the tubular cellular processes of salt and water transfer or to an indirect effect upon tubular function mediated by release of adrenal cortical hormones or neurogenic activity. The change in urine flow may reflect a secondary change in the production of the antidiuretic hormone $(\mathrm{ADH})$ by the posterior pituitary. The possibility that local renal hemodynamic adjustments also play an important role cannot be excluded. The study reported in this paper was un-

1 This study was supported by a grant from the American Heart Association.

2 These data have appeared in preliminary form elsewhere (1).

3 Fellow of the Life Insurance Research Fund.

4 Present address: Walter Reed Army Medical Center, Washington, D. C.

5 Present address: Boston City Hospital, Boston, Mass. dertaken in an attempt to resolve these uncertainties regarding the mechanism of the renal functional response to $l$-epinephrine and $l$-norepinephrine.

\section{METHODS}

Renal clearance methods were used to evaluate renal hemodynamics and to measure the urinary excretion of sodium and potassium during the response to the intravenous infusion of $l$-epinephrine, $l$-norepinephrine, ephedrine, angiotonin, and S-methyl isothiourea sulfate in twenty-two apparently normal human subjects. They ranged in age from 17 to 62 years, were almost equally divided as to sex, and were clinically free from cardiovascular and renal disease. In addition, the renal functional response to $l$-norepinephrine was determined in two patients with Addison's disease, one patient who had had a bilateral adrenalectomy for carcinoma of the prostate, and one patient with diabetes insipidus. All subjects received a regular hospital diet containing about $10 \mathrm{Gm}$. sodium chloride daily.

These studies were made in the morning with the subjects in the basal state, resting quietly in bed. Each subject had ingested 500 to $1000 \mathrm{ml}$. of water one to two hours prior to the study. Saline solution was not administered; all infusions were made up in 5 per cent dextrose in water immediately before using in order to avoid the depression in $\mathrm{p}$-aminohippurate extraction noted by Baldwin, Schreiner, Breed, Wesson, and Maxwell (10) when $\mathrm{PAH}$ and glucose are incubated together in distilled water for several hours.

The renal clearance of sodium p-aminohippurate $(\mathrm{PAH})^{6}$ or Diodrast ${ }^{\circledR}$ was employed in measuring the effective renal plasma flow. Since the hematocrit values did not change appreciably these values may be equated with the renal blood flow. Inulin clearance served as a measure of glomerular filtration rate. These procedures and the analytical methods employed have been described in detail elsewhere $(11,12)$. All plasma and urine specimens were analyzed for sodium and potassium with an internal standard flame photometer.

${ }^{6}$ We are indebted to Dr. W. Boger and the Medical Research Division of Sharp and Dohme, Inc., Philadelphia, Penna. for a generous supply of sodium p-aminohippurate. 
The same general plan was followed throughout. Three ten-minute "control" clearance periods were obtained. The blood pressure, measured sphygmomanometrically, and pulse rate were recorded at frequent intervals. Upon completion of the control measurements an intravenous infusion of the pressor agent (diluted in 5 per cent dextrose in water) was started. The concentration of pressor substance in the infusion was $8 \mu \mathrm{g}$. per $\mathrm{ml}$. for $l$-norepinephrine, $710 \mathrm{mg}$. per $\mathrm{ml}$. for S-methyl isothiourea sulfate, 2 units per $\mathrm{ml}$. for angiotonin, ${ }^{8}$ and $2 \mathrm{mg}$. per ml. for ephedrine. An attempt was made to achieve a sustained increment of 25 to $50 \mathrm{~mm}$. $\mathrm{Hg}$ in systolic blood pressure. This pressor response was nearly always achieved with the infusion of $l$-norepinephrine regulated at 12 to $15 \mu \mathrm{g}$. per minute, S-methyl isothiourea at $15 \mathrm{mg}$. per minute, angiotonin at 5 units per minute and ephedrine at $5 \mathrm{mg}$. per minute. When the blood pressure had stabilized for ten to fifteen minutes, the bladder was emptied, washed out with distilled water and the urine discarded. Three ten-minute "pressor"

7 Supplied through the courtesy of the Medical Research Department of Winthrop Stearns, Inc., New York, as Levophed $\$$.

8 Generously provided for this study by Dr. Oscar Helmer and the Lilly Laboratory for Clinical Research, Indianapolis, Indiana. clearance periods were then collected. During the infusion of pressor drug, blood pressure and pulse rate were recorded every two minutes. The infusion of the drug was discontinued and ten to fifteen minutes were allowed for equilibration. The urine obtained during this period was discarded and three ten-minute "recovery" clearance periods were obtained. Again, the blood pressure and pulse rate were measured frequently (at least once each minute).

Two normal subjects were infused with $l$-epinephrine 9 in 5 per cent dextrose $(2 \mu \mathrm{g}$. per $\mathrm{ml})$. The infusions were maintained at a rate of $2 \mu \mathrm{g}$. per minute, an amount insufficient to cause a rise in systemic blood pressure or obvious peripheral circulatory effect.

In four normal subjects high spinal anesthesia ( $\mathrm{T} 8$ to T4) was induced by the intrathecal instillation of $7.2 \mathrm{mg}$. of nupercaine. Under the continued influence of high spinal anesthesia, three control renal clearance periods were obtained. The effective renal plasma flow was measured by the clearance of Diodrast ${ }^{\circ}$. The renal hemodynamic response and electrolyte and water excretion during administration of $l$-norepinephrine were then observed for two or three additional periods. Only values for those clearance periods obtained when the level of

${ }^{9} \mathrm{We}$ are indebted to Dr. M. M. Rapport for a supply of pure $l$-epinephrine.

TABLE I

The effect of l-norepinephrine on renal function in adrenal cortical insufficiency *

\begin{tabular}{|c|c|c|c|c|c|c|c|c|c|c|c|c|c|}
\hline \multicolumn{3}{|c|}{ Subject } & \multirow[b]{2}{*}{ Procedure } & \multirow[b]{2}{*}{$\underset{m m . \dot{H} g}{\text { B.P. }}$} & \multirow[b]{2}{*}{$\begin{array}{l}\text { P.R. } \\
\text { min. }\end{array}$} & \multirow[b]{2}{*}{$\underset{m l . / m i n}{\mathrm{~V}}$} & \multirow[b]{2}{*}{$\underset{m l . / m i n}{\mathrm{GFR}}$} & \multirow[b]{2}{*}{$\underset{m l . / m i n}{\mathrm{RPF}}$} & \multirow[b]{2}{*}{$\underset{\%}{\mathrm{FF}}$} & \multirow[b]{2}{*}{$\underset{\mu E q . / \min .}{\mathrm{U}_{\mathrm{Na}} \mathrm{V}}$} & \multirow[b]{2}{*}{$\underset{\mu E q . / m i n}{\mathrm{UkV}}$} & \multirow[b]{2}{*}{$\mathrm{C}_{\mathrm{N}} / \mathrm{C}_{\text {in }}$} & \multirow[b]{2}{*}{$\underset{\%}{\mathrm{C}_{\mathrm{K} / \mathrm{C}_{\mathrm{ir}}}}$} \\
\hline Sex & Age & S.A. & & & & & & & & & & & \\
\hline \multicolumn{14}{|c|}{ Addison's disease } \\
\hline F & $\begin{array}{c}\text { R. S. } \\
38\end{array}$ & 1.84 & $\begin{array}{l}\text { Control } \\
\text { Infusion } \\
\text { Recovery }\end{array}$ & $\begin{array}{l}146 / 86 \\
184 / 120 \\
138 / 90\end{array}$ & $\begin{array}{l}74 \\
62 \\
76\end{array}$ & $\begin{array}{l}5.2 \\
5.7 \\
2.2\end{array}$ & $\begin{array}{l}110 \\
100 \\
105\end{array}$ & $\begin{array}{l}450 \\
305 \\
425\end{array}$ & $\begin{array}{l}24 \\
33 \\
25\end{array}$ & $\begin{array}{r}406.0 \\
72.6 \\
113.9\end{array}$ & $\begin{array}{l}57.7 \\
34.5 \\
26.9\end{array}$ & $\begin{array}{l}2.7 \\
0.5 \\
0.8\end{array}$ & $\begin{array}{r}13.6 \\
8.8 \\
6.6\end{array}$ \\
\hline $\mathbf{M}$ & $\begin{array}{c}\text { S. G. } \\
43\end{array}$ & 1.70 & $\begin{array}{l}\text { Control } \\
\text { Infusion } \\
\text { Recovery }\end{array}$ & $\begin{array}{l}112 / 76 \\
168 / 110 \\
114 / 72\end{array}$ & $\begin{array}{l}68 \\
56 \\
80\end{array}$ & $\begin{array}{l}1.7 \\
2.5 \\
1.4\end{array}$ & $\begin{array}{l}95 \\
90 \\
75\end{array}$ & $\begin{array}{l}360 \\
260 \\
335\end{array}$ & $\begin{array}{l}26 \\
34 \\
23\end{array}$ & $\begin{array}{r}254.6 \\
63.7 \\
141.6\end{array}$ & $\begin{array}{l}52.6 \\
45.0 \\
24.1\end{array}$ & $\begin{array}{l}2.1 \\
0.5 \\
1.4\end{array}$ & $\begin{array}{r}14.5 \\
13.0 \\
8.3\end{array}$ \\
\hline \multicolumn{14}{|c|}{ After bilateral adrenalectomy } \\
\hline $\mathbf{M}$ & $\begin{array}{c}\text { J. J. } \\
58\end{array}$ & 1.68 & $\begin{array}{l}\text { Control } \\
\text { Infusion } \\
\text { Recovery }\end{array}$ & $\begin{array}{l}126 / 76 \\
170 / 90 \\
126 / 74\end{array}$ & $\begin{array}{l}80 \\
64 \\
80\end{array}$ & $\begin{array}{l}3.3 \\
3.0 \\
5.6\end{array}$ & $\begin{array}{l}90 \\
85 \\
90\end{array}$ & $\begin{array}{l}510 \\
295 \\
455\end{array}$ & $\begin{array}{l}18 \\
22 \\
20\end{array}$ & $\begin{array}{r}146.6 \\
57.3 \\
115.5\end{array}$ & $\begin{array}{l}56.7 \\
33.0 \\
24.7\end{array}$ & $\begin{array}{l}1.2 \\
0.5 \\
0.9\end{array}$ & $\begin{array}{r}12.7 \\
7.8 \\
5.5\end{array}$ \\
\hline
\end{tabular}

* All values are averages of three determinations. Infusions of $l$-norepinephrine were administered throughout the "infusion" period at a rate sufficient to produce the desired pressor response. The arterial blood pressure (B.P.) was measured sphygmomanometrically. Abbreviations are as follows:

S.A. = body surface $\left(\mathrm{M}^{2}\right)$

P.R. = pulse rate

$\mathrm{V}=$ urine flow (ml./min.)

$\mathrm{GFR}=$ glomerular filtration rate, inulin clearance $(\mathrm{ml} . / \mathrm{min}$.

$\mathrm{RPF}=$ renal plasma flow, sodium $\mathrm{p}$-aminohippurate clearance $(\mathrm{ml} . / \mathrm{min}$.)

$\mathrm{FF}=$ filtration fraction-GFR/RPF (\%)

$\mathrm{U}_{\mathrm{Na}} \mathrm{V}=$ urinary sodium output $(\mu \mathrm{Eq} . / \mathrm{min}$.

$\mathrm{U}_{\mathbf{K}} \mathrm{V}=$ urinary potassium output $(\mu \mathrm{Eq} . / \mathrm{min}$.

$\mathrm{C}_{\mathrm{Na}} / \mathrm{C}_{\mathrm{in}}=$ sodium-inulin clearance ratio or proportion of filtered sodium excreted $(\%)$

$\mathbf{C}_{\mathrm{K}} / \mathbf{C}_{\mathrm{in}}=$ potassium-inulin clearance ratio or proportion of filtered potassium excreted (\%). 
TABLE II

Effect of l-norepinephrine on renal function in a patient with diabetes insipidus and in normal subjects during Pitressin (@induced antidiuresis*

\begin{tabular}{|c|c|c|c|c|c|c|c|c|c|c|c|c|c|}
\hline \multicolumn{3}{|c|}{ Subject } & \multirow[b]{2}{*}{ Procedure } & \multirow[b]{2}{*}{$\underset{m m . \dot{H} g}{\text { B.P }}$} & \multirow[b]{2}{*}{$\begin{array}{l}\text { P.R. } \\
\text { min. }\end{array}$} & \multirow[b]{2}{*}{$\stackrel{\mathrm{V}}{\mathrm{V} . / \min }$} & \multirow[b]{2}{*}{$\underset{m l . / m i n .}{\mathrm{GFR}}$} & \multirow[b]{2}{*}{$\underset{m l . / m i n .}{\mathrm{RPF}}$} & \multirow[b]{2}{*}{$\underset{\%}{\mathrm{FF}}$} & \multirow[b]{2}{*}{$\underset{\mu E q . / m i n}{\mathrm{UNaV}_{\mathrm{Na}}}$} & \multirow[b]{2}{*}{$\underset{\mu E q . / m i n .}{U K V}$} & \multirow[b]{2}{*}{$\underset{\%}{\mathrm{C}_{\mathrm{Na}} / \mathrm{C}_{\mathrm{in}}}$} & \multirow[b]{2}{*}{$\begin{array}{c}\mathrm{C}_{\mathrm{K}} / \mathrm{C}_{\mathrm{in}} \\
\mathrm{F}\end{array}$} \\
\hline Sex & Age & S.A. & & & & & & & & & & & \\
\hline \multicolumn{14}{|c|}{ Diabetes insipidus ( $l$-norepinephrine) } \\
\hline $\mathbf{F}$ & $\begin{array}{c}\text { D. A. } \\
35\end{array}$ & 1.78 & $\begin{array}{l}\text { Control } \\
\text { Infusion } \\
\text { Recovery }\end{array}$ & $\begin{array}{l}126 / 78 \\
154 / 90 \\
114 / 72\end{array}$ & $\begin{array}{l}70 \\
66 \\
74\end{array}$ & $\begin{array}{r}12.3 \\
8.7 \\
12.5\end{array}$ & $\begin{array}{l}150 \\
125 \\
155\end{array}$ & $\begin{array}{l}585 \\
430 \\
600\end{array}$ & $\begin{array}{l}26 \\
29 \\
26\end{array}$ & $\begin{array}{r}218.6 \\
52.6 \\
132.9\end{array}$ & $\begin{array}{l}68.5 \\
57.3 \\
33.8\end{array}$ & $\begin{array}{l}1.1 \\
0.3 \\
0.6\end{array}$ & $\begin{array}{r}10.3 \\
10.4 \\
4.8\end{array}$ \\
\hline \multicolumn{14}{|c|}{ Normal subjects during Pitressin $®$ antidiuresis ( $l$-norepinephrine) } \\
\hline $\mathbf{M}$ & P. M. & 1.72 & $\begin{array}{l}\text { Control } \\
\text { Pitressin } ® \\
\text { Pit. + l-nor. } \\
\text { Recovery }\end{array}$ & $\begin{array}{l}116 / 74 \\
116 / 76 \\
144 / 90 \\
130 / 76\end{array}$ & $\begin{array}{l}64 \\
64 \\
60 \\
60\end{array}$ & $\begin{array}{r}12.3 \\
0.8 \\
0.8 \\
0.4\end{array}$ & $\begin{array}{l}150 \\
155 \\
155 \\
160\end{array}$ & $\begin{array}{l}690 \\
630 \\
485 \\
530\end{array}$ & $\begin{array}{l}22 \\
25 \\
32 \\
30\end{array}$ & $\begin{array}{l}31.6 \\
31.9 \\
24.1 \\
25.2\end{array}$ & $\begin{array}{l}42.8 \\
44.3 \\
36.8 \\
36.7\end{array}$ & $\begin{array}{l}0.2 \\
0.2 \\
0.1 \\
0.1\end{array}$ & $\begin{array}{l}6.8 \\
6.8 \\
5.4 \\
5.4\end{array}$ \\
\hline $\mathbf{F}$ & $\begin{array}{c}\text { J. M. } \\
38\end{array}$ & 1.37 & $\begin{array}{l}\text { Control } \\
\text { Pitressin }{ }^{\circledR} \\
\text { Pit. }+l \text {-nor. }\end{array}$ & $\begin{array}{l}104 / 64 \\
108 / 62 \\
152 / 92\end{array}$ & $\begin{array}{l}84 \\
82 \\
68\end{array}$ & $\begin{array}{l}5.0 \\
1.8 \\
0.8\end{array}$ & $\begin{array}{l}105 \\
100 \\
105\end{array}$ & $\begin{array}{l}475 \\
500 \\
460\end{array}$ & $\begin{array}{l}22 \\
20 \\
24\end{array}$ & $\begin{array}{l}46.3 \\
43.1 \\
22.6\end{array}$ & $\begin{array}{r}13.5 \\
14.7 \\
8.5\end{array}$ & $\begin{array}{l}0.3 \\
0.3 \\
0.3\end{array}$ & $\begin{array}{l}3.8 \\
6.3 \\
4.7\end{array}$ \\
\hline $\mathbf{F}$ & R. S. & 1.57 & $\begin{array}{l}\text { Control } \\
\text { Pitressin } ® \\
\text { Pit. }+l \text {-nor. } \\
\text { Recovery }\end{array}$ & $\begin{array}{l}112 / 72 \\
104 / 74 \\
152 / 100 \\
110 / 72\end{array}$ & $\begin{array}{l}92 \\
84 \\
56 \\
80\end{array}$ & $\begin{array}{l}6.6 \\
0.9 \\
0.7 \\
0.7\end{array}$ & $\begin{array}{r}95 \\
95 \\
90 \\
140\end{array}$ & $\begin{array}{l}490 \\
515 \\
310 \\
610\end{array}$ & $\begin{array}{l}20 \\
24 \\
30 \\
24\end{array}$ & $\begin{array}{l}51.3 \\
65.2 \\
22.2 \\
51.4\end{array}$ & $\begin{array}{l}34.6 \\
34.6 \\
25.0 \\
29.3\end{array}$ & $\begin{array}{l}0.4 \\
0.4 \\
0.2 \\
0.3\end{array}$ & $\begin{array}{l}8.9 \\
6.4 \\
6.8 \\
5.3\end{array}$ \\
\hline $\mathbf{M}$ & J. R. & 1.92 & $\begin{array}{l}\text { Control } \\
\text { Pitressin } \AA \\
\text { Pit. +l-nor. } \\
\text { Recovery }\end{array}$ & $\begin{array}{l}120 / 74 \\
122 / 80 \\
166 / 102 \\
126 / 76\end{array}$ & $\begin{array}{l}68 \\
68 \\
54 \\
74\end{array}$ & $\begin{array}{l}5.5 \\
1.4 \\
1.3 \\
1.5\end{array}$ & $\begin{array}{l}140 \\
145 \\
130 \\
180\end{array}$ & $\begin{array}{l}590 \\
525 \\
400 \\
650\end{array}$ & $\begin{array}{l}24 \\
27 \\
32 \\
25\end{array}$ & $\begin{array}{l}304.0 \\
304.8 \\
197.5 \\
440.5\end{array}$ & $\begin{array}{r}102.6 \\
83.6 \\
39.3 \\
48.0\end{array}$ & $\begin{array}{l}1.6 \\
1.5 \\
1.1 \\
2.1\end{array}$ & $\begin{array}{r}16.3 \\
11.3 \\
6.8 \\
7.0\end{array}$ \\
\hline \multicolumn{14}{|c|}{ Normal subjects ( $l$-norepinephrine + Pitressin $®$ ) } \\
\hline $\mathbf{F}$ & K. N. & 1.46 & $\begin{array}{l}\text { Control } \\
l \text {-norepi. } \\
l \text {-nor. +pit. } \\
\text { Recovery }\end{array}$ & $\begin{array}{l}122 / 62 \\
146 / 82 \\
148 / 86 \\
110 / 62\end{array}$ & $\begin{array}{l}96 \\
66 \\
62 \\
92\end{array}$ & $\begin{array}{r}13.3 \\
7.0 \\
0.6 \\
0.4\end{array}$ & $\begin{array}{r}95 \\
85 \\
80 \\
120\end{array}$ & $\begin{array}{l}490 \\
295 \\
355 \\
755\end{array}$ & $\begin{array}{l}20 \\
29 \\
23 \\
16\end{array}$ & $\begin{array}{l}78.0 \\
29.1 \\
17.3 \\
74.2\end{array}$ & $\begin{array}{l}47.0 \\
28.6 \\
16.2 \\
31.3\end{array}$ & $\begin{array}{l}0.6 \\
0.3 \\
0.2 \\
0.5\end{array}$ & $\begin{array}{r}12.4 \\
8.4 \\
5.0 \\
6.6\end{array}$ \\
\hline $\mathbf{F}$ & E. B. & 1.71 & $\begin{array}{l}\text { Control } \\
\text { l-norepi. } \\
\text { l-nor. +pit. } \\
\text { Recovery }\end{array}$ & $\begin{array}{l}102 / 66 \\
138 / 92 \\
144 / 90 \\
100 / 68\end{array}$ & $\begin{array}{l}82 \\
66 \\
62 \\
80\end{array}$ & $\begin{array}{l}7.8 \\
8.7 \\
0.5 \\
0.4\end{array}$ & $\begin{array}{l}120 \\
135 \\
105 \\
135\end{array}$ & $\begin{array}{l}500 \\
490 \\
515 \\
720\end{array}$ & $\begin{array}{l}24 \\
27 \\
20 \\
19\end{array}$ & $\begin{array}{l}45.9 \\
83.1 \\
85.6 \\
48.8\end{array}$ & $\begin{array}{l}33.5 \\
27.1 \\
23.3 \\
27.4\end{array}$ & $\begin{array}{l}0.3 \\
0.5 \\
0.6 \\
0.3\end{array}$ & $\begin{array}{l}5.8 \\
4.2 \\
4.6 \\
4.2\end{array}$ \\
\hline F & F.E. & 1.92 & $\begin{array}{l}\text { Control } \\
l \text {-norepi. } \\
l \text {-nor. +pit. } \\
\text { Recovery }\end{array}$ & $\begin{array}{l}112 / 74 \\
146 / 88 \\
150 / 86 \\
106 / 68\end{array}$ & $\begin{array}{l}86 \\
78 \\
78 \\
92\end{array}$ & $\begin{array}{l}5.7 \\
6.0 \\
0.4 \\
0.6\end{array}$ & $\begin{array}{l}120 \\
105 \\
115 \\
125\end{array}$ & $\begin{array}{l}540 \\
470 \\
445 \\
580\end{array}$ & $\begin{array}{l}20 \\
23 \\
26 \\
22\end{array}$ & $\begin{array}{r}107.1 \\
92.8 \\
66.2 \\
75.7\end{array}$ & $\begin{array}{l}69.4 \\
33.5 \\
27.4 \\
23.9\end{array}$ & $\begin{array}{l}0.6 \\
0.6 \\
0.5 \\
0.4\end{array}$ & $\begin{array}{r}14.4 \\
7.5 \\
6.0 \\
4.8\end{array}$ \\
\hline
\end{tabular}

* All values are for the last of the determinations during the control periods, both infusion periods and the recovery periods, with the exception of the patient with diabetes insipidus (D. A.) in which the values are the usual averages of three determinations in each phase.

anesthesia was at T8 or higher have been included in this study.

Pitressin (1 milliunit per $\mathrm{kg}$. per hour) was administered intravenously to seven normal subjects. Following completion of the usual control periods in four subjects, the infusion of Pitressin was started and three additional clearance periods were obtained. An infusion of $l$-norepinephrine was then given in addition for the next three periods. In three subjects after the usual control periods this procedure was reversed; i.e., the effect of $l$-norepinephrine alone was followed for three periods and then during the action of Pitressin ${ }^{\circledR}$ for three addi- tional clearance periods. In all seven subjects recovery was observed in the usual manner upon the termination of the infusions.

\section{RESULTS}

The findings are set out in Tables $\mathrm{I}$ to $\mathrm{V}$ and in the figures. All values appearing in the Tables are averages of three successive determinations except where noted. In Figures 4 to 6 , the values for electrolyte and water excretion are expressed as percentages of the averaged control figures in 
TABLE III

The effect of l-norepinephrine on renal function in normal subjects during high spinal anesthesia*

\begin{tabular}{|c|c|c|c|c|c|c|c|c|c|c|c|c|}
\hline \multicolumn{3}{|c|}{ Subject } & \multirow[b]{2}{*}{ Procedure } & \multirow[b]{2}{*}{$\underset{m m . \dot{H} g}{\text { B.P. }}$} & \multirow[b]{2}{*}{$\begin{array}{l}\text { P.R. } \\
\text { min. }\end{array}$} & \multirow[b]{2}{*}{$\underset{m l . / m i n}{\mathrm{~V}}$} & \multirow[b]{2}{*}{$\underset{m l . / m i n .}{\text { GFR }}$} & \multirow[b]{2}{*}{$\underset{m l . / m i n .}{\mathrm{RPF}}$} & \multirow[b]{2}{*}{$\begin{array}{l}\text { FF } \\
\%\end{array}$} & & \multirow[b]{2}{*}{${ }_{\%}^{\mathrm{C}_{\mathrm{Na}} / \mathrm{C}_{\text {in }}}$} & \multirow[b]{2}{*}{$\underset{\%}{\mathrm{C}_{\mathrm{K}} / \mathrm{C}_{\mathrm{in}}}$} \\
\hline Sex & Age & $\underset{M^{2}}{\text { S.A. }}$ & & & & & & & & $\underset{\mu E q . / m i n}{U_{\mathrm{NaV}}}$ & & \\
\hline
\end{tabular}

Normal subjects during high spinal anesthesia ( $l$-norepinephrine)

\begin{tabular}{|c|c|c|c|c|c|c|c|c|c|c|c|c|c|}
\hline $\mathbf{M}$ & $\underset{31}{\mathrm{~J} . \mathrm{G} .}$ & 1.60 & $\begin{array}{l}\text { Sp. anes. (T4) } \\
\text { Sp. }+l \text {-nor. }\end{array}$ & $\begin{array}{l}102 / 68 \\
132 / 90\end{array}$ & $\begin{array}{l}72 \\
48\end{array}$ & $\begin{array}{l}0.5 \\
0.4\end{array}$ & $\begin{array}{l}85 \\
85\end{array}$ & $\begin{array}{l}460 \\
360\end{array}$ & $\begin{array}{l}19 \\
23\end{array}$ & $\begin{array}{l}55.7 \\
21.0\end{array}$ & $\begin{array}{l}55.6 \\
45.4\end{array}$ & $\begin{array}{l}0.5 \\
0.2\end{array}$ & $\begin{array}{l}17.5 \\
15.5\end{array}$ \\
\hline $\mathbf{M}$ & A. L. & 1.96 & $\begin{array}{l}\text { Sp. anes. (T7) } \\
\text { Sp. }+l \text {-nor. }\end{array}$ & $\begin{array}{l}108 / 60 \\
138 / 82\end{array}$ & $\begin{array}{l}88 \\
60\end{array}$ & $\begin{array}{l}0.9 \\
0.4\end{array}$ & $\begin{array}{l}115 \\
100\end{array}$ & $\begin{array}{l}540 \\
400\end{array}$ & $\begin{array}{l}23 \\
25\end{array}$ & $\begin{array}{r}135.4 \\
34.1\end{array}$ & $\begin{array}{l}61.1 \\
38.9\end{array}$ & $\begin{array}{l}0.8 \\
0.3\end{array}$ & $\begin{array}{r}11.4 \\
9.5\end{array}$ \\
\hline $\mathbf{M}$ & $\mathrm{W}_{31} \mathrm{~N}$. & 2.03 & $\begin{array}{l}\text { Sp. anes. (T7) } \\
\text { Sp. }+l \text {-nor. }\end{array}$ & $\begin{array}{l}112 / 62 \\
128 / 82\end{array}$ & $\begin{array}{l}86 \\
58\end{array}$ & $\begin{array}{l}0.6 \\
0.7\end{array}$ & $\begin{array}{l}105 \\
110\end{array}$ & $\begin{array}{l}610 \\
540\end{array}$ & $\begin{array}{l}17 \\
21\end{array}$ & $\begin{array}{l}47.5 \\
19.9\end{array}$ & $\begin{array}{l}28.6 \\
32.9\end{array}$ & $\begin{array}{l}0.3 \\
0.1\end{array}$ & $\begin{array}{l}6.8 \\
7.3\end{array}$ \\
\hline $\mathbf{M}$ & C. I. & 1.78 & $\begin{array}{l}\text { Sp. anes. (T8)* } \\
\text { Sp. }+l \text {-nor.* }\end{array}$ & $\begin{array}{l}114 / 72 \\
140 / 90\end{array}$ & $\begin{array}{l}72 \\
58\end{array}$ & $\begin{array}{l}0.3 \\
0.6\end{array}$ & $\begin{array}{l}95 \\
95\end{array}$ & $\begin{array}{l}345 \\
335\end{array}$ & $\begin{array}{l}27 \\
28\end{array}$ & $\begin{array}{l}16.9 \\
19.5\end{array}$ & $\begin{array}{l}23.4 \\
38.1\end{array}$ & $\begin{array}{l}0.1 \\
0.2\end{array}$ & $\begin{array}{r}7.7 \\
12.5\end{array}$ \\
\hline
\end{tabular}

* All values are averages of three determinations except in instances denoted with an asterisk. Control clearance periods were obtained during spinal anesthesia. An infusion of $l$-norepinephrine was administered during the continued influence of the spinal anesthesia. The lowest thoracic level of spinal anesthesia during the entire procedure is noted, and no values were accepted when anesthesia level was lower than T8. Abbreviations are the same as in Table I.

order to make possible comparison of the changes produced by the experimental procedure and to minimize for this purpose the variation between individuals attributable to differences in dietary preparation, appetite, and other uncontrolled variables. Plasma sodium and potassium concentrations were not significantly altered in any of these studies and are not included in the tables.

In most instances, $l$-norepinephrine was used in preference to $l$-epinephrine, because the unpredictable side-effects and variable response to $l$-epinephrine make it much less safe to use than $l$-norepinephrine. However, both agents produce the same changes in renal functions and it may be inferred that the findings reported apply to the adrenal medullary hormones in general.
Changes observed in renal hemodynamics and in the urinary excretion of sodium, potassium and water in response to the adrenal medullary hormones

1. During adrenal cortical insufficiency: The response to intravenous $l$-norepinephrine was observed in two patients with Addison's disease. One (R. S.) was receiving DOCA (pellets, 450 mg., implanted 6 months previously) and the other (S. G.) was maintained on cortisone $(12.5 \mathrm{mg}$. daily-last dose 15 hours prior to study) and DOCA (pellets, $300 \mathrm{mg}$., implanted 2 weeks previously). In both patients, the renal response to $l$-norepinephrine was typical of that reported in normal subjects (7). The averaged figures for

TABLE IV

The effect of minimal amounts of l-epinephrine on renal function in normal subjects *

\begin{tabular}{|c|c|c|c|c|c|c|c|c|c|c|c|c|c|}
\hline \multicolumn{3}{|c|}{ Subject } & \multirow[b]{2}{*}{ Procedure } & \multirow[b]{2}{*}{$\underset{m m .}{\mathrm{B} . P} \dot{H} g$} & \multirow[b]{2}{*}{$\begin{array}{l}\text { P.R. } \\
\text { min. }\end{array}$} & \multirow[b]{2}{*}{$\underset{m l . / m i n}{\mathrm{~V}}$} & \multirow[b]{2}{*}{$\underset{m l . / m i n .}{\mathrm{GFR}}$} & \multirow[b]{2}{*}{$\underset{m l . / m i n}{\mathrm{RPF}}$} & \multirow[b]{2}{*}{$\underset{\%}{\mathrm{FF}}$} & \multirow[b]{2}{*}{$\underset{\mu E q . / \min .}{\mathrm{UN}_{\mathrm{N}_{\mathrm{a}} \mathrm{V}}}$} & \multirow[b]{2}{*}{$\underset{\mu E q . / \min .}{\mathrm{UkV}_{\mathrm{K}}}$} & \multirow[b]{2}{*}{$\underset{\%}{\mathrm{C}_{\mathrm{Na}} / \mathrm{C}_{\mathrm{in}}}$} & \multirow[b]{2}{*}{$\mathrm{C}_{\%} / \mathrm{C}_{\mathrm{in}}$} \\
\hline Sex & Age & S.A. & & & & & & & & & & & \\
\hline \multicolumn{14}{|c|}{ Normal subjects (minimal amounts of $l$-epinephrine) } \\
\hline $\mathbf{M}$ & $\begin{array}{c}\text { A. } \mathrm{H} \text {. } \\
33\end{array}$ & 1.90 & $\begin{array}{l}\text { Control } \\
\text { Infusion } \\
\text { Recovery }\end{array}$ & $\begin{array}{l}102 / 68 \\
108 / 66 \\
108 / 66\end{array}$ & $\begin{array}{l}68 \\
68 \\
68\end{array}$ & $\begin{array}{l}5.6 \\
9.5 \\
4.2\end{array}$ & $\begin{array}{l}150 \\
150 \\
150\end{array}$ & $\begin{array}{l}780 \\
640 \\
700\end{array}$ & $\begin{array}{l}20 \\
24 \\
21\end{array}$ & $\begin{array}{l}84.7 \\
58.1 \\
59.1\end{array}$ & $\begin{array}{r}102.0 \\
62.6 \\
35.0\end{array}$ & $\begin{array}{l}0.4 \\
0.3 \\
0.3\end{array}$ & $\begin{array}{r}15.1 \\
9.3 \\
5.3\end{array}$ \\
\hline $\mathbf{F}$ & $\begin{array}{c}\text { M. K. } \\
44\end{array}$ & 1.76 & $\begin{array}{l}\text { Control } \\
\text { Infusion } \\
\text { Recovery }\end{array}$ & $\begin{array}{l}112 / 72 \\
114 / 70 \\
114 / 70\end{array}$ & $\begin{array}{l}76 \\
76 \\
76\end{array}$ & $\begin{array}{l}6.9 \\
5.7 \\
1.8\end{array}$ & $\begin{array}{r}95 \\
100 \\
110\end{array}$ & $\begin{array}{l}625 \\
450 \\
505\end{array}$ & $\begin{array}{l}15 \\
22 \\
22\end{array}$ & $\begin{array}{r}134.8 \\
92.9 \\
110.0\end{array}$ & $\begin{array}{l}54.1 \\
32.1 \\
25.7\end{array}$ & $\begin{array}{l}1.0 \\
0.7 \\
0.7\end{array}$ & $\begin{array}{r}14.8 \\
8.3 \\
6.1\end{array}$ \\
\hline
\end{tabular}

* All values are averages of three determinations. Peripheral circulatory change was avoided by infusion of $l$-epinephrine in an amount insufficient to cause pressor response or change in pulse rate. Abbreviations as in Table I. 
TABLE $v$

The effects of angiotonin, S-methyl isothiourea sulfate and ephedrine on renal function and urinary electrolyte excretion in normal human subjects *

\begin{tabular}{|c|c|c|c|c|c|c|c|c|c|c|c|c|c|}
\hline \multicolumn{3}{|c|}{ Subject } & \multirow[b]{2}{*}{ Procedure } & \multirow[b]{2}{*}{$\underset{m m . \mathbf{H g}}{\text { B.P. }}$} & \multirow[b]{2}{*}{$\begin{array}{l}\text { P.R. } \\
\text { min. }\end{array}$} & \multirow[b]{2}{*}{$\underset{m l . / m i n}{\mathrm{~V}}$} & \multirow[b]{2}{*}{$\underset{m l . / m i n}{\text { GFR }}$} & \multirow[b]{2}{*}{$\underset{m l . / m i n}{\mathrm{RPF}}$} & \multirow[b]{2}{*}{$\underset{\%}{\mathrm{FF}}$} & \multirow[b]{2}{*}{$\underset{\mu E q . / \min }{\mathrm{U}_{\mathrm{Na}}}$} & \multirow[b]{2}{*}{$\underset{\mu E q . / m i n}{U_{k V}}$} & \multirow[b]{2}{*}{$\mathrm{C}_{\mathrm{Na} / \mathrm{C}_{\text {in }}}$} & \multirow[b]{2}{*}{$\underset{\%}{\mathrm{Ck}_{\boldsymbol{K}} / \mathrm{C}_{\text {in }}}$} \\
\hline Sex & Age & S.A. & & & & & & & & & & & \\
\hline \multicolumn{14}{|c|}{ (Angiotonin) } \\
\hline $\mathbf{M}$ & $\begin{array}{c}\text { F. } Z \text {. } \\
48\end{array}$ & 1.96 & $\begin{array}{l}\text { Control } \\
\text { Infusion } \\
\text { Recovery }\end{array}$ & $\begin{array}{l}136 / 78 \\
152 / 94 \\
128 / 80\end{array}$ & $\begin{array}{l}72 \\
72 \\
72\end{array}$ & $\begin{array}{l}9.2 \\
1.7 \\
4.4\end{array}$ & $\begin{array}{l}145 \\
120 \\
130\end{array}$ & $\begin{array}{l}665 \\
400 \\
590\end{array}$ & $\begin{array}{l}22 \\
31 \\
22\end{array}$ & $\begin{array}{l}485.0 \\
122.9 \\
195.4\end{array}$ & $\begin{array}{l}78.6 \\
64.1 \\
86.1\end{array}$ & $\begin{array}{l}2.3 \\
0.7 \\
1.0\end{array}$ & $\begin{array}{l}13.3 \\
13.0 \\
16.3\end{array}$ \\
\hline $\mathbf{M}$ & $\begin{array}{c}\text { E. H. } \\
44\end{array}$ & 1.92 & $\begin{array}{l}\text { Control } \\
\text { Infusion } \\
\text { Recovery }\end{array}$ & $\begin{array}{l}138 / 84 \\
170 / 106 \\
132 / 82\end{array}$ & $\begin{array}{l}64 \\
58 \\
68\end{array}$ & $\begin{array}{l}7.3 \\
1.1 \\
2.7\end{array}$ & $\begin{array}{r}105 \\
65 \\
95\end{array}$ & $\begin{array}{l}495 \\
235 \\
460\end{array}$ & $\begin{array}{l}21 \\
27 \\
21\end{array}$ & $\begin{array}{r}248.7 \\
50.1 \\
135.9\end{array}$ & $\begin{array}{l}82.9 \\
28.3 \\
78.4\end{array}$ & $\begin{array}{l}1.7 \\
0.7 \\
1.1\end{array}$ & $\begin{array}{r}14.7 \\
9.2 \\
15.8\end{array}$ \\
\hline F & $\begin{array}{c}\text { B. E. } \\
29\end{array}$ & 1.43 & $\begin{array}{l}\text { Control } \\
\text { Infusion } \\
\text { Recovery }\end{array}$ & $\begin{array}{l}108 / 70 \\
150 / 86 \\
104 / 70\end{array}$ & $\begin{array}{l}84 \\
72 \\
84\end{array}$ & $\begin{array}{l}4.9 \\
1.4 \\
6.9\end{array}$ & $\begin{array}{r}115 \\
95 \\
135\end{array}$ & $\begin{array}{l}600 \\
365 \\
640\end{array}$ & $\begin{array}{l}19 \\
26 \\
21\end{array}$ & $\begin{array}{r}127.4 \\
23.2^{\circ} \\
94.9\end{array}$ & $\begin{array}{l}53.1 \\
30.3 \\
42.4\end{array}$ & $\begin{array}{l}0.8 \\
0.2 \\
0.5\end{array}$ & $\begin{array}{r}10.2 \\
7.3 \\
6.9\end{array}$ \\
\hline \multicolumn{14}{|c|}{ (S-methyl isothiourea) } \\
\hline $\mathbf{M}$ & $\begin{array}{c}\text { P. C. } \\
24\end{array}$ & 1.65 & $\begin{array}{l}\text { Control } \\
\text { Infusion }\end{array}$ & $\begin{array}{l}128 / 72 \\
140 / 86\end{array}$ & $\begin{array}{l}64 \\
50\end{array}$ & $\begin{array}{l}4.5 \\
3.7 \\
\end{array}$ & $\begin{array}{r}130 \\
95\end{array}$ & $\begin{array}{l}695 \\
435\end{array}$ & $\begin{array}{l}19 \\
22\end{array}$ & $\begin{array}{l}74.5 \\
29.5\end{array}$ & $\begin{array}{l}51.4 \\
36.0\end{array}$ & $\begin{array}{l}0.4 \\
0.2\end{array}$ & $\begin{array}{r}10.1 \\
9.3\end{array}$ \\
\hline$F$ & $\begin{array}{c}\text { G. M. } \\
28\end{array}$ & 1.35 & $\begin{array}{l}\text { Control } \\
\text { Infusion } \\
\text { Recovery }\end{array}$ & $\begin{array}{l}108 / 70 \\
130 / 92 \\
126 / 88\end{array}$ & $\begin{array}{l}76 \\
56 \\
60\end{array}$ & $\begin{array}{l}1.6 \\
0.7 \\
0.5\end{array}$ & $\begin{array}{l}80 \\
40 \\
40\end{array}$ & $\begin{array}{l}340 \\
140 \\
160\end{array}$ & $\begin{array}{l}24 \\
30 \\
25\end{array}$ & $\begin{array}{l}56.6 \\
14.7 \\
20.7\end{array}$ & $\begin{array}{r}21.9 \\
10.5 \\
9.7\end{array}$ & $\begin{array}{l}0.5 \\
0.3 \\
0.4\end{array}$ & $\begin{array}{l}6.4 \\
6.2 \\
6.0\end{array}$ \\
\hline \multicolumn{14}{|c|}{ (Ephedrine) } \\
\hline $\mathrm{F}$ & $\begin{array}{c}\text { M. T. } \\
28\end{array}$ & 1.66 & $\begin{array}{l}\text { Control } \\
\text { Infusion } \\
\text { Recovery }\end{array}$ & $\begin{array}{l}102 / 58 \\
128 / 64 \\
132 / 60\end{array}$ & $\begin{array}{l}76 \\
88 \\
86 \\
\end{array}$ & $\begin{array}{l}4.9 \\
4.5 \\
1.3 \\
\end{array}$ & $\begin{array}{l}125 \\
135 \\
130\end{array}$ & $\begin{array}{l}595 \\
585 \\
585\end{array}$ & $\begin{array}{l}21 \\
23 \\
23\end{array}$ & $\begin{array}{r}51.2 \\
113.7 \\
91.4\end{array}$ & $\begin{array}{l}61.4 \\
53.6 \\
24.4 \\
\end{array}$ & $\begin{array}{l}0.3 \\
0.6 \\
0.5\end{array}$ & $\begin{array}{r}10.7 \\
8.8 \\
4.2\end{array}$ \\
\hline $\mathbf{M}$ & $\begin{array}{c}\text { E. P. } \\
29\end{array}$ & 1.96 & $\begin{array}{l}\text { Control } \\
\text { Infusion } \\
\text { Recovery }\end{array}$ & $\begin{array}{l}142 / 84 \\
176 / 90 \\
170 / 88\end{array}$ & $\begin{array}{l}80 \\
86 \\
88\end{array}$ & $\begin{array}{l}4.9 \\
4.6 \\
6.3\end{array}$ & $\begin{array}{l}125 \\
125 \\
135\end{array}$ & $\begin{array}{l}520 \\
590 \\
650\end{array}$ & $\begin{array}{l}23 \\
21 \\
20\end{array}$ & $\begin{array}{l}249.3 \\
253.2 \\
285.9\end{array}$ & $\begin{array}{l}67.7 \\
62.5 \\
58.0\end{array}$ & $\begin{array}{l}1.4 \\
1.4 \\
1.6\end{array}$ & $\begin{array}{r}11.8 \\
10.9 \\
9.8\end{array}$ \\
\hline $\mathrm{F}$ & $\begin{array}{c}\text { A. G. } \\
55\end{array}$ & 1.66 & $\begin{array}{l}\text { Control } \\
\text { Infusion } \\
\text { Recovery }\end{array}$ & $\begin{array}{l}116 / 72 \\
144 / 72 \\
136 / 68\end{array}$ & $\begin{array}{l}60 \\
70 \\
70\end{array}$ & $\begin{array}{r}10.3 \\
9.3 \\
6.1\end{array}$ & $\begin{array}{l}75 \\
80 \\
85\end{array}$ & $\begin{array}{l}335 \\
350 \\
345\end{array}$ & $\begin{array}{l}22 \\
23 \\
24\end{array}$ & $\begin{array}{l}182.2 \\
291.3 \\
237.7\end{array}$ & $\begin{array}{l}39.2 \\
34.1 \\
23.9\end{array}$ & $\begin{array}{l}1.7 \\
2.6 \\
2.0\end{array}$ & $\begin{array}{r}11.0 \\
9.0 \\
6.0\end{array}$ \\
\hline $\mathbf{M}$ & $\begin{array}{c}\text { R. W. } \\
17\end{array}$ & 1.83 & $\begin{array}{l}\text { Control } \\
\text { Infusion } \\
\text { Recovery }\end{array}$ & $\begin{array}{l}120 / 78 \\
164 / 86 \\
156 / 84\end{array}$ & $\begin{array}{l}64 \\
76 \\
80\end{array}$ & $\begin{array}{l}3.4 \\
8.9 \\
3.0\end{array}$ & $\begin{array}{l}120 \\
110 \\
115\end{array}$ & $\begin{array}{l}555 \\
590 \\
565\end{array}$ & $\begin{array}{l}21 \\
19 \\
20\end{array}$ & $\begin{array}{l}130.5 \\
202.7 \\
243.0\end{array}$ & $\begin{array}{l}98.1 \\
86.5 \\
42.9\end{array}$ & $\begin{array}{l}0.9 \\
1.4 \\
1.5\end{array}$ & $\begin{array}{r}20.7 \\
19.1 \\
9.5\end{array}$ \\
\hline
\end{tabular}

* All values are averages of three determinations.

Abbreviations are the same as in Table I.

the effective renal plasma flow (RPF), glomerular filtration rate (GFR), urinary sodium excretion $\left(\mathrm{U}_{\mathrm{Na}} \mathrm{V}\right)$, and urinary potassium excretion $\left(U_{K} V\right)$ during the control periods, during the $l$-norepinephrine infusion, and during the recovery periods are presented in Table I. Renal plasma flow (RPF) decreased during the infusion of norepinephrine. The urinary excretion of sodium $\left(\mathrm{U}_{\mathrm{Na}} \mathrm{V}\right)$ and potassium $\left(\mathrm{U}_{\mathbf{K}} \mathrm{V}\right)$ declined sharply while glomerular filtration rate (GFR) did not appear to change. Urine volume (V) tended to increase during the pressor period and fell abruptly when $l$-norepinephrine was discon- tinued. During the recovery periods the effective renal plasma flow returned essentially to control values and urinary sodium output tended to increase, but potassium excretion continued to fall. The changes in systemic and renal hemodynamics, urinary sodium, and potassium excretion and urine volume in one (S. G.) of these patients with Addison's disease are shown in Figures 1 and 2.

One patient (J. J.) with carcinoma of the prostate, who had undergone a total bilateral adrenalectomy (maintained on cortisone- $35 \mathrm{mg}$. dailylast dose 12 hours prior to study) was infused with $l$-norepinephrine in the usual manner. The 


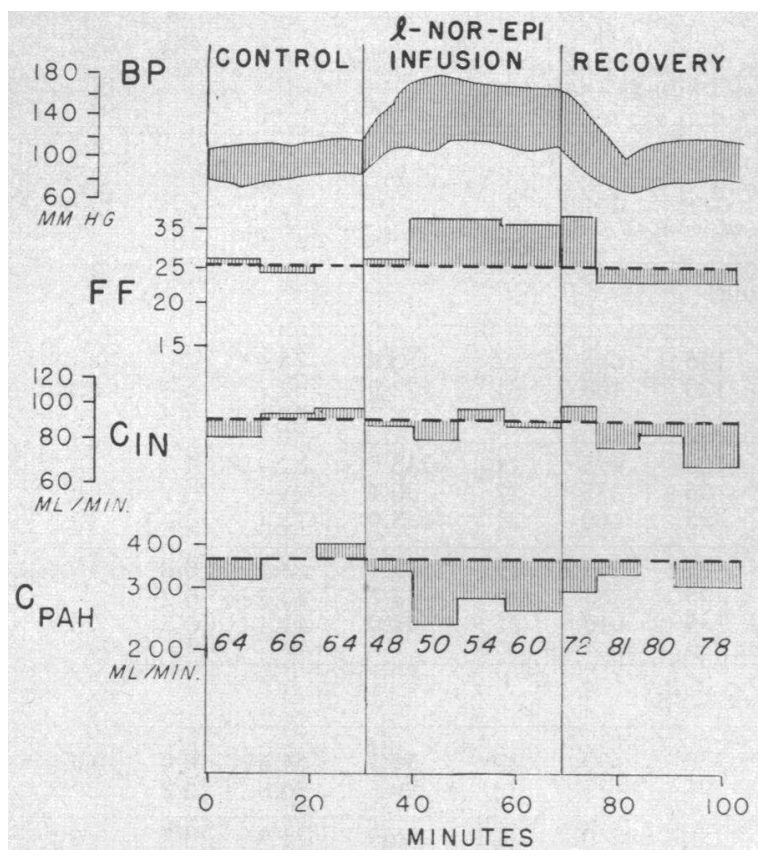

Fig. 1. The Effect of L-Norepinephrine on Filtration Rate and Renal Plasma Flow in a Patient with Addison's Disease (Subject S. G.)

Blood pressure (BP-sphygmomanometer), glomerular filtration rate $\left(\mathrm{C}_{\mathbf{i n}}\right.$-inulin clearance), renal plasma flow ( $\mathrm{C}_{\mathrm{pah}}-\mathrm{p}$-amino-hippurate clearance), filtration fraction $\left(\mathrm{FF}-\mathrm{C}_{\mathrm{in}} / \mathrm{C}_{\text {pah }} \times 100\right)$ and pulse rate (numerical values inserted below $C_{\text {pah }}$ ) were measured before, during, and after intravenous infusion of $l$-norepinephrine. All values except those for blood pressure and pulse rate are plotted semi-logarithmically against time. Renal plasma flow and filtration fraction rose with the elevation in blood pressure during the infusion and returned to the control levels during recovery. The values just after beginning and stopping the infusion of pressor agent have been omitted in computing the averages for this patient (S. G.) in Table I because intra-renal delay and the process of equilibration resulted in unreliable clearances.

decrements in renal plasma flow and urinary excretion of sodium and potassium (Table I) were similar to those reported in normal subjects (7), and to those observed in patients with Addison's disease maintained on adequate substitution therapy. The renal plasma flow promptly returned toward control values when the $l$-norepinephrine infusion was stopped. Sodium excretion in the urine tended to increase but urinary potassium excretion continued to decrease during the recovery periods. The change in water excretion was atypical since urine flow did not fall upon termination of the drug infusion, but tended to rise.

2. During anti-diuretic hormone deficiency or excess: When $l$-norepinephrine was administered by intravenous infusion to one patient with wellestablished and untreated diabetes insipidus, the blood pressure increased and the renal plasma flow diminished without a statistically significant change in filtration rate (Table II). Urinary concentration and excretion of sodium and potassium also decreased during the pressor response as in normal persons, but urine flow fell by 20 per cent on the average. During recovery, the renal plasma flow promptly increased, sodium output

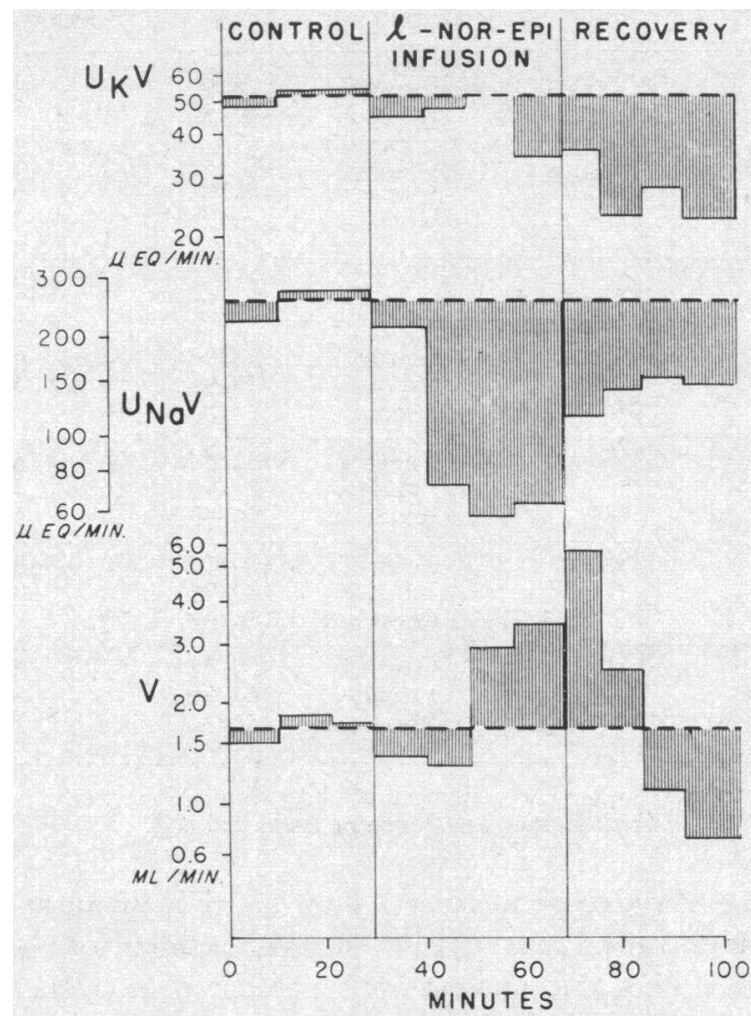

Fig. 2. The Effect of L-Norepinephrine on Electrolyte and Water Excretion in a Patient with Adpison's Disease (Subject S. G.)

Values for potassium $\left(U_{K} V\right)$, sodium $\left(U_{N_{a}} V\right)$, and water excretion (V) during the study depicted in Figure 1 are plotted semi-logarithmically against time. Electrolyte excretion diminished while urine flow increased. The fall in potassium excretion was delayed and persisted into the recovery period, whereas the changes in sodium and water output tended to return to control values. 
rose moderately, but potassium excretion fell still more.

Pitressin ${ }^{\circledR}$ ( 1 milliunit per kg. per hour) had no statistically significant effect upon blood pressure, pulse rate, renal plasma flow or glomerular filtration rate in four normal subjects. Since determinations made during the period of equilibration were not discarded in these studies, only the last values obtained in successive periods during each phase (control, Pitressin ${ }^{\circledR}$, or l-norepinephrine infusion, Pitressin ${ }^{\circledR}$ and $l$-norepinephrine infusion, and recovery) are set out in Table II in order to provide for a closer approach to the "steady" state. The urine flow always decreased markedly without much change in electrolyte excretion, as others (13) have found. However, sodium output increased by 13 per cent in one subject (R. S.) and potassium excretion decreased by 18 per cent in another (J. R.). When $l$-norepinephrine was administered intravenously after the antidiuretic effect of Pitressin ${ }^{\circledR}$ was well-established and maintained, no change in urine flow occurred and anti-diuresis persisted throughout recovery. The renal plasma flow fell and electrolyte output decreased without change in filtration in every instance. Thus, with the exception of antidiuresis, Pitressin ${ }^{\circledR}$ did not alter the response to $l$-norepinephrine in any significant manner (Figure 3 ). Likewise, $l$-norepinephrine did not affect the response to Pitressin ${ }^{\circledR}$ in three subjects in whom pressor and renal functional effects of $l$-norepinephrine were established prior to administration of Pitressin ${ }^{\circledR}$ (Table II). In one of these, E. B. (Table II), sodium output increased in association with more or less unchanging renal plasma flow under the influence of $l$-norepinephrine. This anomalous response persisted during the anti-diuretic action of Pitressin ${ }^{\circledR}$. During recovery after both drugs were withdrawn the urine flow remained depressed and all other values tended to return to control levels. The potassium output continued to fall in F. E. (Table II), however.

3. During high spinal anesthesia: Spinal anesthesia, with loss of sensation up to T8 or higher, caused no statistically significant fall in arterial blood pressure in four normal subjects. Renal plasma flow and filtration rate were unaffected, as others (14) have reported. When $l$-norepinephrine was infused, the usual decrease in renal

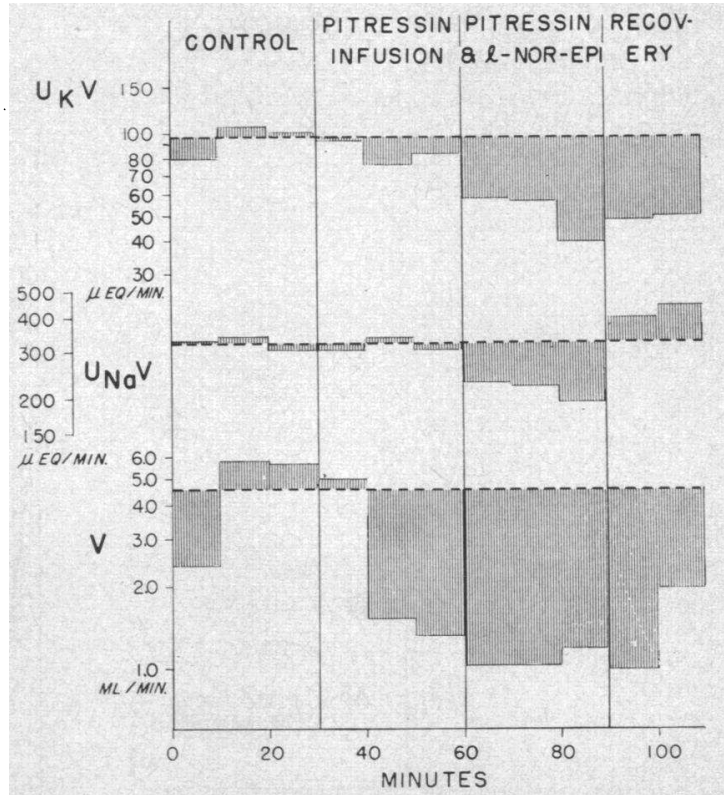

Fig. 3. The Effect of L-Norepinephrine on Electrolyte and Water Excretion during Pitressin Antidiuresis (Subject J. R.)

Infusion of Pitressin ${ }^{\circledR}$ produced no significant change in potassium $\left(\mathrm{U}_{\mathrm{K}} \mathrm{V}\right)$ and sodium $\left(\mathrm{U}_{\mathrm{Na}} \mathrm{V}\right)$ excretion, but urine flow (V) fell markedly. During $l$-norepinephrine infusion both potassium and sodium output decreased without change in urine flow.

plasma flow (Diodrast ${ }^{\circledR}$ clearance) (Table III) occurred in all but one (C. I.) while glomerular filtration rate remained unchanged in all. $L$-norepinephrine caused no consistent change in urine flow. The urinary excretion of sodium decreased in all but one subject (C. I.), who presented unusually low values for sodium output during the control periods. Urinary potassium excretion decreased in two (J. G. and A. L.), increased in one (C. I.), and did not change significantly in the other subject (W. N.). It is noteworthy that in C. I., $l$-norepinephrine failed to reduce sodium and potassium output in association with a failure to diminish renal plasma flow. In general, however, high spinal anesthesia did not interfere with the usual changes in renal functional responses to $l$-norepinephrine.

4. In the absence of a pressor response to l-epinephrine: When $l$-epinephrine ${ }^{10}$ was administered

\footnotetext{
${ }^{10} \mathrm{~L}$-epinephrine was chosen for this study, because $l$-norepinephrine, even in minute dosage, caused at least some degree of peripheral vasoconstriction and arterial hypertension.
} 


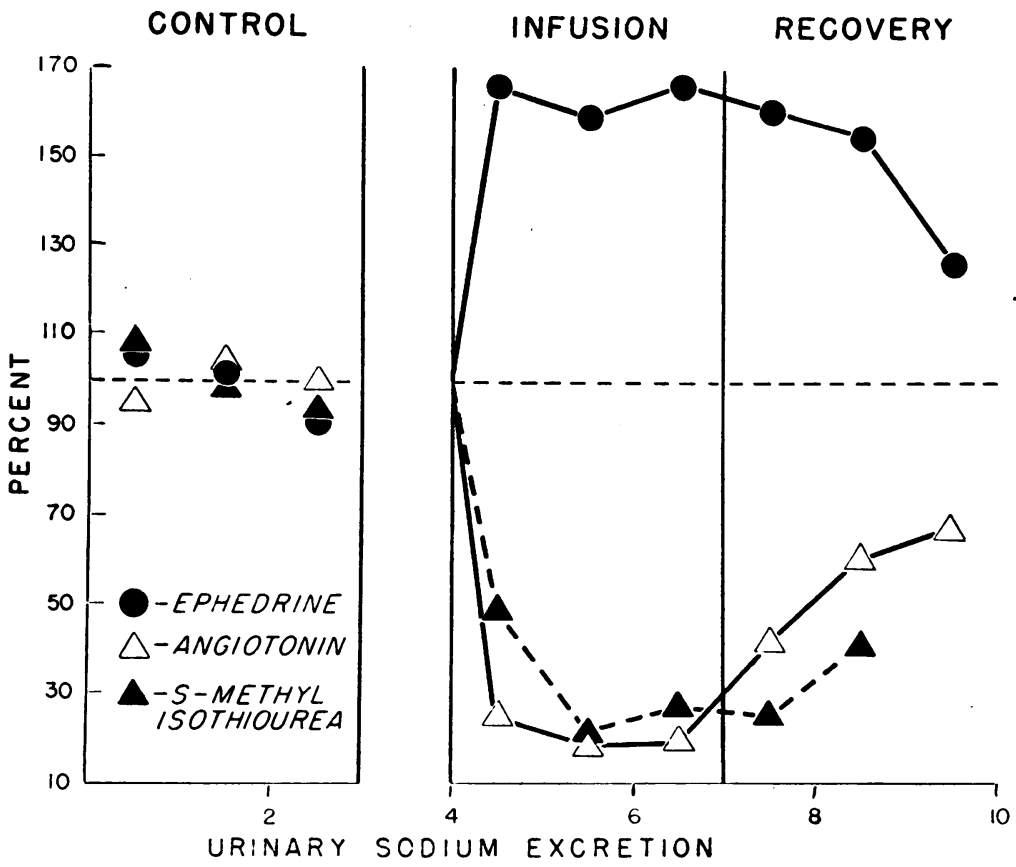

Fig. 4. The Effect of Ephedrine, Angiotonin, and S-Methyi lsothiourea Sulfate on Urine Flow

The values for urine flow during each "period" (usually 10 to 15 minutes in duration) have been averaged and expressed here as the percentage of the pooled and averaged control figures. An infusion of ephedrine (closed circles), angiotonin (open triangles), or S-methyl isothiourea sulfate was administered intravenously during the periods designated "Infusion." In each study values were obtained during three periods in phase (control, infusion, and recovery); urine collected during the period immediately after starting the infusion was discarded. See Table $\mathrm{V}$ for further information on the studies collated here. The urine flow was usually rising during the control periods owing to preliminary ingestion of water.

In subjects receiving ephedrine the peak of the diuretic response was reached shortly after the infusion of ephedrine was started; the urine flow decreased sharply thereafter and stabilized at values at approximately 70 per cent of control on the average. With infusion of angiotonin and S-methyl isothiourea sulfate, urine flow fell abruptly, tending to return to control values on recovery from angiotonin and remaining depressed after S-methyl isothiourea.

intravenously at a rate of $2 \mu \mathrm{g}$. per minute to two normal subjects, neither arterial blood pressure nor pulse rate changed significantly and skin pallor did not develop.

Despite the apparent lack of peripheral circulatory adjustment during the $l$-epinephrine infusion, effective renal plasma flow decreased and the urinary excretion of both sodium and potassium fell sharply (Table IV). Glomerular filtration rate did not change significantly. In one subject (A. H.) urine flow increased strikingly during the drug infusion and remained relatively unchanged in the other subject (M. K.). When $l$-epinephrine was discontinued the urine flow in both subjects fell abruptly. During recovery the urinary sodium excretion tended to return toward control values in both subjects, but urinary potassium excretion continued to decrease despite the return of renal plasma flow toward control levels. These changes did not differ appreciably from those observed in response to $l$-epinephrine or $l$-norepinephrine when arterial pressure increased (7). 
Comparison of changes in renal hemodynamics and in urinary excretion of water, sodium, and potassium during the action of other pressor agents

1. Angiotonin and $S$-methyl isothiourea: In order to assess the role of renal hemodynamic adjustments in producing the change in urine formation during the action of the adrenal medullary hormones, the response to other agents having a similar action was studied. For this purpose, angiotonin and S-methyl isothiourea sulfate were chosen, because it is known $(15,16)$ that both induce renal vasoconstriction and systemic hemodynamic alterations similar to those produced by $l$-norepinephrine. Neither substance is chemically similar to the medullary hormones, and they would seem the less likely, therefore, to behave as analogues in bringing about changes through direct action upon the tubular cells.

Angiotonin ( 5 units per minute) was administered intravenously to three, and S-methyl iso- thiourea sulfate (15 mg. per minute) to two normal individuals. Both agents raised the arterial pressure and reduced the pulse rate in association with renal vasoconstriction. In every instance renal plasma flow and glomerular filtration rate decreased (Table V) with a rise in the filtration fraction.

The urine flow always decreased, returning to or toward control levels during recovery from the action of angiotonin and remaining depressed following S-methyl isothiourea sulfate (Figure 4). The decrement in water excretion was relatively greater than the fall in filtration and electrolyte excretion during the infusion of angiotonin, so that the urinary concentration of electrolyte and inulin increased, indicating a relative increment in water reabsorption. S-methyl isothiourea sulfate, on the other hand, depressed urine flow no more than filtration and much less than electrolyte excretion.

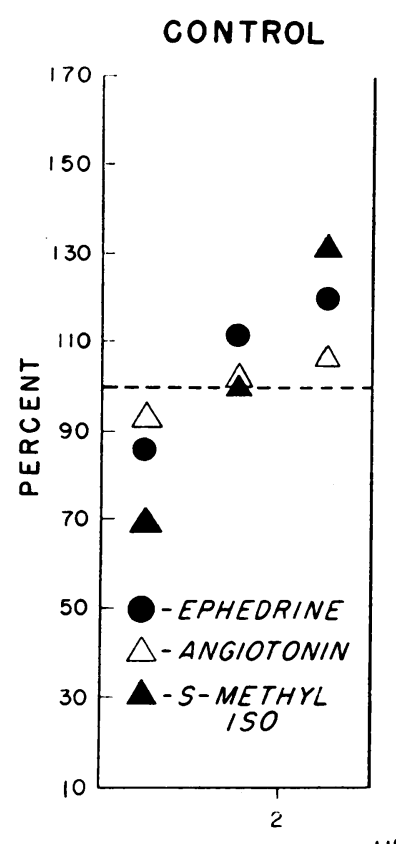

\section{INFUSION RECOVERY}

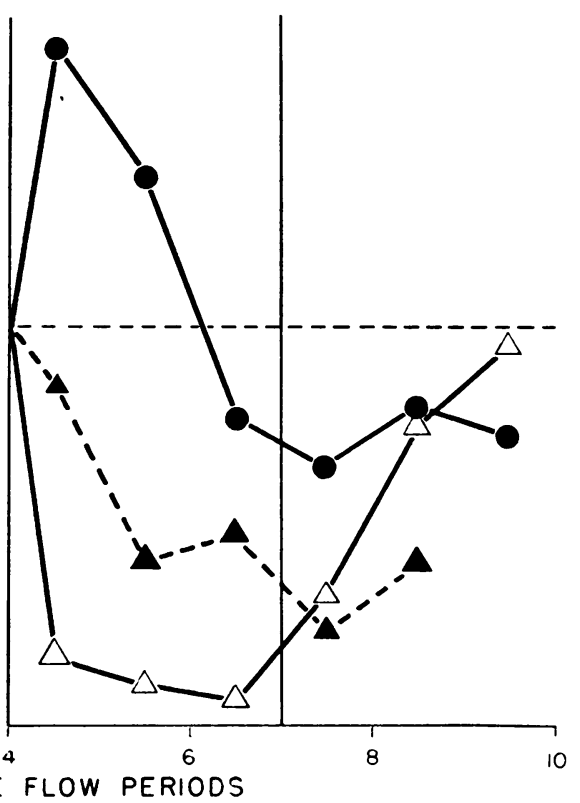

Fig. 5. The Effect of Ephedrine, Angiotonin, and S-Methyl Isothiourea Sulfate on Urinary Sodium Excretion

The data for urinary sodium excretion in the subjects presented in Figure 4 are treated here in a similar fashion. Sodium excretion decreased markedly during infusion of angiotonin and S-methyl isothiourea sulfate, and increased by 70 per cent on the average during ephedrine infusion. There was a tendency to return toward the control values during recovery in all instances. 
CONTROL

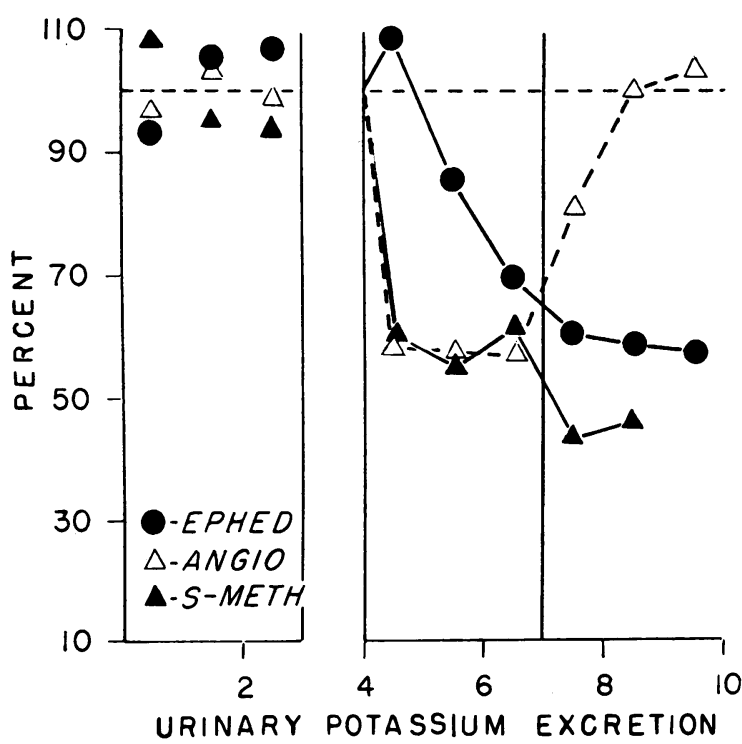

Fig. 6. The Effect of Ephedrine, Angiotonin, and S-Methyl Isothiourea Sulfate on Urinary PotasSIUM EXCRETION

The data are handled as in Figures 4 and 5. Potassium excretion declined during the pressor action of all three agents, returned to the control values after angiotonin and remained depressed during recovery after ephedrine and S-methyl isothiourea.

Sodium and potassium output (Figures 5 and 6 ) fell off markedly during the action of both drugs. Sodium clearance decreased more than filtration, i.e., the sodium-inulin clearance ratio fell (Table $\mathrm{V})$, whereas potassium clearance tended to decrease to relatively the same extent as, or somewhat more than filtration. All values tended to rise during the recovery period.

These changes indicate a relative increase in tubular reabsorption of electrolytes, but owing to the fact that filtration usually decreased greatly, the actual quantities of sodium, potassium, and water reabsorbed by the tubules diminished.

2. Ephedrine: The effect of ephedrine upon urine function was investigated in four normal persons (Table V) in order to determine whether a substance chemically analogous to $l$-epinephrine would produce similar changes in function. Ephedrine is known to affect the cardiovascular system in more or less the same manner as $l$-epinephrine, but its effect on renal hemodynamics is uncertain. Ranges and Bradley (5) observed re- nal ischemia in normal human subjects following administration of single doses of ephedrine (45 to $50 \mathrm{mg}$. subcutaneously). In subsequent studies Maxwell, Morales, and Crowder (17) were unable to detect any change in renal plasma flow following similar doses of the drugs. Their findings have been confirmed in the course of the studies reported here (Table V). Neither renal plasma flow nor glomerular filtration changed, except to increase slightly, in any subject during infusion of ephedrine ( $2 \mathrm{mg}$. per min.). Since the arterial pressure rose, the renal vascular resistance must have increased proportionately.

The changes in urine formation during the pressor action of ephedrine differed from those produced by $l$-epinephrine and $l$-norepinephrine. The averaged urine flow did not change in three subjects, but rose markedly in one. During the "recovery" period, urine flow decreased in two, rose in one, and returned to the control value in one study (Table V, Figure 4). Sodium output always increased, in two studies remaining elevated and in two decreasing during recovery. Since filtration did not change, the sodium-inulin clearance ratio rose, indicating decreased tubular reabsorption (Table V, Figure 5). The tubular reabsorption of potassium appeared to increase, since potassium output and the potassium-inulin clearance ratio decreased (Table V, Figure 6).

\section{DISCUSSION}

The extent, rapidity, and variety of the physiologic changes observed during intravenous infusion of $l$-epinephrine and $l$-norepinephrine make analysis of their mode of action exceedingly difficult. Both agents probably act directly upon tissues throughout the body. Nonetheless, indirect secondary effects may play a more prominent role in determining the total physiologic response. This consideration is particularly important in an evaluation of the changes in kidney function. It seems reasonable to attribute intra-renal vasoconstriction to direct stimulation of the arteriolar musculature by the circulating hormone, but the changes in urine formation could be ascribed to secondary release of other humoral agents, to neurogenic activity, or to local hemodynamic adjustments. 
The results of this study indicate clearly that the retention of sodium and potassium during the pressor action of $l$-norepinephrine (and presumably, also, of $l$-epinephrine) does not require the active intervention of the adrenal cortex. The typical response was observed in patients with Addison's disease or after bilateral adrenalectomy. Although these patients were maintained on adequate substitution therapy, the pathology or absence of the adrenals precluded active adrenocortical participation in the response to norepinephrine.

A change in the production of antidiuretic hormone by the posterior pituitary may account for the alteration in water excretion. Tubular reabsorption of electrolytes is believed to be iso-osmotic (18) and the increased electrolyte reabsorption induced by $l$-norepinephrine should have entailed a corresponding increment in water reabsorption. However, urine flow remained unchanged or actually increased. Although the additional fluid administered during the infusion of $l$-norepinephrine may have contributed, it is likely that suppression of $\mathrm{ADH}$ secretion by the action of the medullary hormone played an important role under these circumstances (19). Thus, urine flow decreased in association with the decrement in electrolyte excretion in a patient with diabetes insipidus (Table II). It may be inferred that inability to suppress further the elaboration of $\mathrm{ADH}$ "unmasked" the potentially anti-diuretic effect of increased tubular electrolyte reabsorption. In contrast, in a patient with Addison's disease who drank a liter of water without a diuretic response just prior to the study illustrated in Figures 1 and 2 , the urine flow increased only during the infusion of $l$-norepinephrine. Since $l$-norepinephrine did not block the antidiuretic response to Pitressin ${ }^{\circledR}$, this phenomenon suggests that a reduction in $\mathrm{ADH}$ formation corrected temporarily the impaired diuretic response of Addison's disease (20). The oliguria usually observed following withdrawal of $l$-norepinephrine or $l$-epinephrine could be accounted for by excessive release of ADH following the period of suppression. $L$-norepinephrine elicited a fall in sodium and potassium output without change in urine flow during antidiuresis imposed by preliminary administration of Pitressin ${ }^{\circledR}$, strongly suggesting that the changes in electrolyte and water reabsorption occur independently.
The kidney receives a rich nerve supply from the autonomic nervous system through which the changes in tubule function and the renal circulation could have been mediated (18). Spinal anesthesia to levels sufficiently high to interrupt the neural outflow to the kidney did not affect the response to the usual doses of $l$-norepinephrine. ${ }^{11}$ Although it is possible that denervation was not complete under these circumstances, even a partial effect should have interfered with the response to some extent if it were neurogenically mediated. Despite successful maintenance of the arterial pressure more or less unchanged, all these patients were incapable of appropriate adjustment to postural change so that the blood pressure tended to fall, indicating widespread loss of splanchnic autonomic activity in which the kidney probably participated. Activation of an intra-renal neural network by the direct action of $l$-norepinephrine circulating in the blood cannot be excluded.

Distention of the renal vasculature and stimulation of local stretch receptors by the elevation in arterial pressure might have also set in train local readjustments. However, $l$-epinephrine produced the usual alterations in electrolyte excretion even when administered in doses too small to change the blood pressure. When hypertension developed during the infusion of angiotonin or S-methyl isothiourea the renal functional response did not altogether resemble that produced by the medullary hormones. Sodium and potassium output diminished but the change could have been attributed to the concomitant fall in glomerular filtration. Reabsorption of sodium and potassium did increase relative to filtration but total reabsorption diminished. The pressor response to ephedrine was associated with an even more marked divergence in the pattern of renal functional response. Neither renal blood flow nor glomerular filtration changed significantly, and the output of sodium actually increased. It may be concluded, therefore, that the local pressor response per se was of little or no importance in determining the alteration in renal function.

Renal ischemia, on the other hand, appeared to be invariably associated with diminished sodium output. Angiotonin, S-methyl isothiourea sulfate,

11 All these patients were oliguric during the control periods presumably owing to release of antidiuretic hormone. 
$l$-epinephrine, and $l$-norepinephrine all induced vasoconstrictive renal ischemia and a reduction in sodium excretion. When ischemia did not develop despite intra-renal vasoconstriction (as in subjects E. B. and C. I. [Tables II and III] during administration of $l$-norepinephrine) or during the action of ephedrine, sodium excretion remained unchanged or increased. These facts suggest that reduction of the blood flow to tubular tissue may affect sodium reabsorptive activity. Pullman and McClure (21) failed to find any significant change in sodium excretion in six normal young men during infusion of $l$-norepinephrine, despite marked reduction in renal blood flow. It is possible that administration of isotonic saline, the smaller doses of the drug employed throughout the better part of each experiment, and the short period of study at dosage levels comparable to those reported here may account for the absence of a change in sodium output. The specific changes that modify potassium excretion remain obscure. The output of potassium was reduced, not only during the pressor action of each drug, but also when blood pressure did not change. The only common factor in these diverse responses appeared to be intra-renal vasoconstriction.

It is quite possible that the adrenal medullary hormones act directly upon renal tubular cells to accelerate electrolyte reabsorption. These substances are highly active and are known (22) to affect various cell types and various intra-cellular activities. The fact that $l$-norepinephrine failed to reduce sodium excretion when the renal blood flow did not decrease is opposed to this possibility. The plasma concentration of norepinephrine may have been too low in these instances but the blood pressure did rise. The response to ephedrine was unexpected and at variance with the hypothesis (23) that it acts only through the intermediation of epinephrine by blocking amine oxidase, thus preventing oxidation of epinephrine. It is possible that the effect of ephedrine on sodium excretion is attributable to one of its metabolites, to insufficient local release of epinephrine, or, as the data suggest, to its failure to induce a reduction in renal blood flow.

\section{SUM M ARY}

This study has confirmed previous observations of diminished sodium and potassium excretion and a tendency to loss of water in the urine during the pressor action of the adrenal medullary hormones in man.

The change in electrolyte excretion is apparently not mediated through adrenal cortical activation. Three subjects with adrenal cortical insufficiency (two with Addison's disease and one after bilateral adrenalectomy) responded to $l$-norepinephrine like normal persons. Diminished formation of antidiuretic hormone may account for sustained or augmented output of a dilute urine during infusion of the medullary amines. In a patient with diabetes insipidus, $l$-norepinephrine depressed both electrolyte and water excretion. Fitressin ${ }^{\circledR}$ evoked a marked reduction in urine flow without changing electrolyte excretion in three normal subjects receiving an infusion of l-norepinephrine. In four subjects administration of $l$-norepinephrine during antidiuresis produced by an infusion of Pitressin ${ }^{\circledR}$ resulted in diminished electrolyte excretion without a change in urine flow, indicating the independence of the changes in electrolyte and water reabsorption by the renal tubules.

Since high spinal anesthesia (T8 or higher) did not interfere with the action of $l$-norepinephrine in four subjects, the response did not seem to be mediated through neural pathways. Nor did the pressor response per se seem necessary. $L$-epinephrine in doses too small to change the arterial pressure or pulse rate evoked the typical renal functional alterations in two subjects. Other pressor agents (angiotonin, S-methyl isothiourea sulfate, and ephedrine) failed to produce exactly the same response. Sodium and potassium output were lowered by angiotonin (three subjects) and S-methyl isothiourea (two subjects) but the glomerular filtration rate and urine flow were also decreased in association with the reduction in renal blood flow. Ephedrine (four subjects) induced renal vasoconstriction in proportion to the elevation in blood pressure so that renal blood flow did not change. The potassium excretion decreased but sodium output rose without change in filtration.

Sodium reabsorption always seemed to increase in association with a fall in renal blood flow. When renal blood flow remained unchanged as in two of the subjects receiving $l$-norepinephrine and in the four subjects receiving ephedrine, the 
pressor response was associated with diminished tubular reabsorption of sodium. Potassium retention, on the other hand, appeared to be correlated only with intrarenal vasoconstriction. It is impossible to decide on the basis of these findings whether $l$-epinephrine and $l$-norepinephrine act directly upon the renal tubular cells, but the data suggest an indirect action mediated through local hemodynamic adjustments.

\section{ACKNOWLEDGMENT}

We wish to express our gratitude to Mrs. Joan Banfield, Mrs. Lottie Klayman, and Mrs. Michaeleen Patterson for technical assistance. We are indebted to Dr. John Fertig for assistance in a statistical analysis of the data.

\section{REFERENCES}

1. Nickel, J. F., Smythe, C. McC., and Bradley, S. E., Effect of pressor agents on renal water and electrolyte excretion in man. Federation Proc., 1952, $11,113$.

2. Goldenberg, M., Pines, K. L., Baldwin, E. deF., Greene, D. G., and Roh, C. E., The hemodynamic response of man to nor-epinephrine and epinephrine and its relation to the problem of hypertension. Am. J. Med., 1948, 5, 792.

3. Barcroft, H., and Konzett, H., On the actions of noradrenaline, adrenaline, and isopropyl noradrenaline on the arterial blood pressure, heart rate, and muscle blood flow in man. J. Physiol, 1949, 110, 194.

4. Chasis, H., Ranges, H. A., Goldring, W., and Smith, H. W., The control of renal blood flow and glomerular filtration in normal man. J. Clin. Invest., 1938, 17, 683.

5. Ranges, H. A., and Bradley, S. E., Systemic and renal circulatory changes following the administration of adrenin, ephedrine and paredrinol to normal man. J. Clin. Invest., 1943, 22, 687.

6. Barnett, A. J., Blacket, R. B., Depoorter, A. E., Sanderson, P. H., and Wilson, G. M., The action of noradrenaline in man and its relation to phæchromocytoma and hypertension. Clin. Sc., 1950, 9, 151.

7. Smythe, C. McC., Nickel, J. F., and Bradley, S. E., The effect of epinephrine (USP), l-epinephrine, and $l$-norepinephrine on glomerular filtration rate, renal plasma flow, and the urinary excretion of sodium, potassium, and water in normal man. J. Clin. Invest., 1952, 31, 499.

8. Jacobson, W. E., Hammarsten, J. F., and Heller, B. I., The effects of adrenaline upon renal func- tion and electrolyte excretion. J. Clin. Invest., 1951, 30, 1503.

9. Smith, H. W., Goldring, W., Chasis, H., Ranges, H. A., and Bradley, S. E., The William Henry Welch Lectures. II. The application of saturation methods to the study of glomerular and tubular function in the human kidney. J. Mt. Sinai Hosp., 1943, 10, 59.

10. Baldwin, D. S., Schreiner, G. E., Breed, E. S., Wesson, L. G., Jr., and Maxwell, M. H., Depression of apparent p-aminohippurate extraction ratio by glucose. J. Clin. Invest., 1950, 29, 614.

11. Goldring, W., and Chasis, H., Hypertension and Hypertensive Disease. New York, The Commonwealth Fund, 1944.

12. Bradley, S. E., Bradley, G. P., Tyson, C. J., Curry, J. J., and Blake, W. D., Renal function in renal diseases. Am. J. Med., 1950, 9, 766.

13. Murphy, R. J. F., and Stead, E. A., Jr., Effects of exogenous and endogenous posterior pituitary antidiuretic hormone on water and electrolyte excretion. J. Clin. Invest., 1951, 30, 1055.

14. Smith, H. W., Rovenstine, E. A., Goldring, W., Chasis, H., and Ranges, H. A., The effects of spinal anesthesia on the circulation in normal, unoperated man with reference to the autonomy of the arterioles, and especially those of the renal circulation. J. Clin. Invest., 1939, 18, 319.

15. Bradley, S. E., and Parker, B., The hemodynamic effects of angiotonin in normal man. J. Clin. Invest., 1941, 20, 715.

16. McGeorge, M., Sherif, M., and Smirk, F. H., Observations on the properties of S-methyl iso-thiourea sulphate with particular reference to the circulatory effects. J. Physiol., 1942, 100, 474.

17. Maxwell, M. H., Morales, P., and Crowder, C. H., Jr., Effect of therapeutic doses of ephedrine on renal clearances in normal man. Proc. Soc. Exper. Biol. \& Med., 1951, 77, 539.

18. Smith, H. W., The Kidney: Structure and Function in Health and Disease. New York, Oxford Univ. Press, 1951.

19. Verney, E. B., Croonian lectures. Antidiuretic hormone and factors which determine its release. Proc. Roy. Soc., London, 1947, B135, 25.

20. Gaunt, R., Birnie, J. H., and Eversole, W. J., Adrenal cortex and water metabolism. Physiol. Rev., 1949, 29, 281.

21. Pullman, T. N., and McClure, W. W., The effect of 1-nor-adrenaline on electrolyte excretion in normal man. J. Lab. \& Clin. Med., 1952, 39, 711.

22. Griffith, F. R., Jr., Fact and theory regarding the calorigenic action of adrenalin. Physiol. Rev., 1951, 31, 151.

23. Gaddum, J. H., The alkaloid ephedrine. Brit. Med. J., 1938, 1, 713. 